\title{
Habilidades em consciência silábica e fonêmica de crianças com fala desviante com e sem intervenção fonoaudiológica
}

\author{
Syllabic and phonemic awareness abilities in children with \\ speech disorder with and without speech therapy intervention
}

\author{
Paula Tavares Marchetti ${ }^{1}$, Carolina Lisbôa Mezzomo², Carla Aparecida Cielo ${ }^{3}$
}

\begin{abstract}
RESUMO
Objetivo: Comparar o desempenho de crianças com desvio fonológico evolutivo (DFE) que receberam intervenção fonoaudiológica com enfoque fonológico e de crianças com DFE que não receberam esta em habilidades de consciência fonológica (CF). Métodos: Foram avaliados 23 sujeitos com diagnóstico de DFE, sendo que 14 crianças fizeram parte do Grupo de Estudo 1 (GE1), que recebeu terapia fonoaudiológica, e as outras nove fizeram parte do Grupo de Estudo 2 (GE2), que ainda não havia recebido fonoterapia. Todos os sujeitos foram submetidos à avaliação fonoaudiológica e da consciência fonológica. Resultados: Das 26 sub-tarefas de CF aplicadas nos grupos de estudo, houve diferença estatisticamente significativa somente na tarefa T7-dissílabas (reversão silábica), confirmando o pior desempenho do GE2 ( $\leq \leq 0,05)$. Porém, na análise das médias houve tendência a um melhor desempenho do GE1 em comparação ao GE2. Apesar desses resultados, mesmo os sujeitos que receberam intervenção fonoaudiológica tiveram desempenhos muito baixos. Conclusões: Houve diferença estatisticamente significante somente na sub-tarefa T7-dissílabas (reversão silábica) entre crianças com DFE que receberam intervenção fonoaudiológica e crianças com DFE que não receberam fonoterapia. Porém, notou-se uma tendência do grupo que recebeu terapia de ter um melhor desempenho nas tarefas de CF do que o grupo que não recebeu.
\end{abstract}

Descritores: Distúrbios da fala/terapia; Transtornos do desenvolvimento da linguagem/terapia; Linguagem infantil; Fonoterapia

\section{INTRODUÇÃO}

Desvio Fonológico Evolutivo (DFE) é um quadro clínico que se caracteriza por alteração no desenvolvimento normal da fala, com desorganizações, inadaptações ou anormalidades do sistema de sons da criança em relação ao sistema linguístico padrão no qual está inserida. Dessa forma, a fala se torna muitas vezes ininteligível, sem que haja comprometimentos orgânicos ${ }^{(1-5)}$.

Já a Consciência Fonológica (CF) pode ser definida como a habilidade de refletir sobre os sons da fala e sua organização na formação das palavras. É a habilidade de analisar a fala

Trabalho realizado no Departamento de Fonoaudiologia da Universidade Federal de Santa Maria - UFSM - Santa Maria (RS), Brasil.

(1) Mestre, Fonoaudióloga clínica da Audioclínica OuveBem - Santa Maria (RS), Brasil.

(2) Doutora, Professora do Departamento de Fonoaudiologia e do Programa de Pós-graduação em Distúrbios da Comunicação Humana da Universidade Federal de Santa Maria - UFSM - Santa Maria (RS), Brasil.

(3) Doutora, Professora do Departamento de Fonoaudiologia e do Programa de Pós-graduação em Distúrbios da Comunicação Humana da Universidade Federal de Santa Maria - UFSM - Santa Maria (RS), Brasil.

Endereço para correspondência: Paula Tavares Marchetti. R. Mal. Floriano Peixoto, 529/301, Centro, Santa Maria (RS), Brasil, CEP: 97010-310. E-mail: paulam_fono@hotmail.com

Recebido em: 7/8/2008; Aceito em: 1/6/2009 explicitamente em seus componentes fonológicos ${ }^{(6-11)}$.

Algumas pesquisas demonstram que a $\mathrm{CF}$ de crianças com DFE se apresenta em um nível inferior ao de crianças com desenvolvimento de fala normal; além disso, crianças com DFE são de risco para apresentarem problemas de aprendizagem $^{(12-15)}$.

Estudos têm mostrado que crianças com DFE são mais suscetíveis a apresentarem déficits no início da educação formal e nas habilidades em CF, sendo então de risco para atraso na aquisição da leitura e da escrita. Isso pode estar relacionado ao fato de que a representação fonológica é a base para a produção da leitura e da escrita ${ }^{(3,9,11-12,14-18)}$.

Com base nos dados apontados pela literatura, levanta-se a hipótese de que as crianças com DFE poderiam apresentar melhora nas habilidades metafonológicas após intervenção usando modelos fonológicos, mas sem a estimulação direta em CF.

A intervenção que utiliza os modelos fonológicos baseia-se no fato de que as dificuldades na organização dos sons na fala são de natureza fonológica, que essa fala constitui um sistema fonológico inadequado e que a terapia fonoaudiológica atuaria como um reorganizador deste sistema ${ }^{(1-2)}$. Assim, o objetivo da terapia fonológica é facilitar a reorganização cognitiva do sistema fonológico da criança com fala desviante, sendo que 
tais mudanças devem ocorrer em nível de representação mental e não apenas em nível articulatório ${ }^{(1)}$.

Partindo dessa hipótese, tem-se como objetivo desta pesquisa comparar o desempenho nas habilidades em $\mathrm{CF}$ entre crianças com DFE que tenham recebido intervenção fonoaudiológica com enfoque fonológico, e crianças com DFE que não tenham recebido a intervenção.

\section{MÉTODOS}

Esta pesquisa faz parte do projeto Comparação da performance nas tarefas de consciência silábica e fonêmica entre crianças com desenvolvimento fonológico normal e desviante", o qual foi aprovado pelo Comitê de Ética em Pesquisa (CEP) da Universidade Federal de Santa Maria (UFSM) sob o número de processo 23081.007155/2006-13.

Os sujeitos pesquisados foram divididos em dois subgrupos de crianças com DFE: o grupo de estudo 1 (GE1) que recebeu terapia fonológica e o grupo de estudo 2 (GE2) de crianças que não receberam intervenção fonológica para contornar o desvio de fala. Os sujeitos foram selecionados no Serviço de Atendimento Fonoaudiológico (SAF) da UFSM, a partir da análise de todos os pacientes atendidos no setor de fala e no setor de triagem fonoaudiológica.

Os candidatos a participar desta pesquisa foram somente os pacientes que tinham diagnóstico de DFE, que iniciaram atendimento fonoaudiológico no ano de 2006 ou que estivessem na lista de espera para atendimento no setor de fala, com idades entre quatro e oito anos.

Como critérios para inclusão nos grupos, consideraramse aptos a participar da pesquisa os sujeitos que: foram autorizados pelos pais ou responsáveis para a participação por meio do Termo de Consentimento Livre e Esclarecido; apresentaram diagnóstico de desvio fonológico de acordo com a literatura; tinham idade entre quatro e oito anos de idade; e frequentavam ou o jardim de infância, ou a pré-escola, ou a $1^{\mathrm{a}}$ série ou a $2^{\mathrm{a}}$ série do Ensino Fundamental.

Os critérios de exclusão foram: alterações evidentes nos aspectos neurológico, cognitivo, psicológico e/ou emocional, bem como alterações audiológicas.

Para se observar tais aspectos, os pacientes que fariam parte do GE foram submetidos a avaliações fonoaudiológicas e complementares. As avaliações complementares consistiram de avaliações audiológica, neurológica e otorrinolaringológica.

Os pacientes passaram por avaliações do sistema estomatognático e avaliação da fala, realizadas pelas respectivas terapeutas das crianças que já estavam em atendimento no setor de fala - alunas do $7^{\circ}$ semestre do curso de Fonoaudiologia que estavam realizando o estágio supervisionado. Essas alunas realizaram as mesmas avaliações no setor de triagem, nas crianças que estavam na lista de espera, com vistas à aplicação dos critérios de inclusão e de exclusão da pesquisa.

$\mathrm{Na}$ avaliação do sistema estomatognático, foram observados os aspectos relacionados à estrutura dos órgãos fonoarticulatórios (lábios, língua, bochechas, palato mole, palato duro, mandíbula e arcada dentária) bem como à postura, ao tônus, à mobilidade e às funções do sistema estomatognático. Essa avaliação foi realizada a fim de se verificar a existência de fatores orgânicos que pudessem impedir a produção correta dos sons da fala, levando à distorção dos fonemas. Para tal avaliação, foi utilizado o protocolo da própria instituição de origem.

A avaliação fonológica, feita por uma das fonoaudiólogas autoras deste trabalho, consistiu da coleta de uma amostra de fala através da nomeação e fala espontânea, realizada com a utilização do instrumento Avaliação Fonológica da Criança $\mathrm{AFC}^{(19)}$. Esse instrumento é constituído por cinco desenhos temáticos (banheiro, cozinha, sala, veículos, e zoológico) e possibilita a elicitação de todos os fones contrastivos do português brasileiro em todas as posições que podem ocorrer em relação à estrutura da sílaba e da palavra.

Os dados foram gravados em formato digital, em mp3 player modelo $1 \mathrm{G}$ da marca Sony ${ }^{\circledR}$ e gravador analógico com fita cassete modelo RQ-L10 da Panasonic $®$, ambos com qualidade de gravação suficiente para a análise perceptual. Após a coleta, os dados foram submetidos à transcrição fonética restrita e à análise contrastiva. A análise contrastiva foi realizada com o objetivo de comparar o sistema da criança com o sistema padrão do adulto, estabelecendo-se o inventário fonológico desviante de cada criança. As distorções fonéticas, como interdentalizações, ceceios entre outros, não foram considerados como erros fonológicos e, por isto, foram transcritas como corretas. É importante referir que a transcrição utilizada foi a restrita, na qual os diacríticos referentes à distorções não aparecem.

A partir desses critérios, formou-se uma amostra de 23 sujeitos, sendo 14 do GE1(com terapia), sendo quatro meninas e dez meninos, com a média de idade de 6,29 anos; e nove do GE2 (sem terapia), sendo três meninas e seis meninos, com média de idade de 6,06 anos. As crianças do GE1 estavam, em média, há quatro meses em terapia (com um ou dois atendimentos semanais, dependendo do caso) quando foram avaliadas. Os modelos terapêuticos usados foram: Modelo de Ciclos Modificado* e Modelo de Pares Mínimos Oposições Máximas**.

O Modelo de Ciclos Modificado prevê a ocorrência de ciclos de tratamento. Cada ciclo tem duração de três semanas, sendo que em cada semana focaliza-se um processo fonológico. Cada sessão tem duração de 50 minutos e acontecem duas vezes por semana. Visando a eliminação de cada processo, são escolhidos dois sons-alvo que sofrem o processo fonológico, e cada um deles é trabalhado durante uma sessão de terapia. O objetivo principal de cada sessão é fazer com que a criança enuncie o maior número de produções corretas do som estimulado em cinco a dez palavras-estímulo selecionadas cuidadosamente.

O Modelo de Oposições Máximas tem como procedimento

\footnotetext{
*Tyler A. Edwards ML, Saxman JH. Clinical application of two phonologically based treatment procedures. J Speech Hear Disord. 1987;52(4):393-409. **A terapia Metaphon é dividida em fases que focalizam um aspecto específico da habilidade metalinguística. O objetivo inicial é facilitar o conhecimento da natureza contrastiva dos fonemas. Em um segundo momento, o objetivo é demonstrar a importância de tais contrastes para a comunicação. Em ambas as fases a natureza contrastiva é trabalhada através da consciência fonológica ${ }^{(1)}$.
} 
básico o contraste de duas palavras que diferem em apenas um fonema. Os fonemas podem diferir quanto ao número de oposições distintivas - número de traços distintivos que diferenciam os fonemas; quanto à natureza das distinções de traços - traços de classes principais ou de classes não principais; e quanto à relação da gramática da criança antes do tratamento - fonemas conhecidos ou desconhecidos da criança ${ }^{(1)}$. A maneira como esses fonemas diferem é fundamental para as mudanças fonológicas que ocorrem durante o tratamento, logo, espera-se que a apresentação de dois fonemas novos que tenham distinção de classe principal e distinção máxima de traços leve a uma mudança fonológica maior $^{(1)}$. Portanto, em nenhum caso foi utilizado o Modelo Metaphon ${ }^{* * *}$.

A escolha do modelo terapêutico utilizado depende do comprometimento do inventário fonológico de cada criança. No grupo estudado, sete crianças foram submetidas ao Modelo de Ciclos Modificado, seis ao Modelo de Pares Mínimos com oposições máximas e uma iniciou com o Modelo de Ciclos e no decorrer do atendimento optou-se pelo Modelo de Pares Mínimos. Foram consideradas dez sessões, como número mínimo de atendimentos, e 40 como número máximo. Assim, nenhuma criança do GE1, no momento das avaliações, tinha passado por um número inferior a dez sessões de terapia, nem tampouco excedeu a 40 sessões de tratamento.

Para a coleta de dados foi realizada a avaliação CF que, nessas crianças, foi realizada por uma das autoras da pesquisa. A CF foi avaliada por meio do Protocolo de Tarefas de Consciência Fonológica ${ }^{(7-8)}$ que possibilita analisar as habilidades metafonológicas em: segmentação de frases em palavras; realismo nominal; detecção de rimas; síntese silábica; segmentação silábica; detecção de sílabas; reversão silábica; exclusão fonêmica; detecção de fonemas; síntese fonêmica; segmentação fonêmica e reversão fonêmica. Porém, neste trabalho, foram analisadas somente as tarefas no nível da sílaba e do fonema, pois estas habilidades têm mais relação com o nível fonológico, justamente o aspecto formal da língua que estaria afetado nas crianças com DFE.

No que se refere à aplicação do protocolo e à avaliação das respostas das crianças, considerou-se para cada resposta correta na primeira tentativa, o valor 2; para cada resposta correta na segunda tentativa, foi atribuído o valor 1; e para as respostas incorretas foi atribuído o valor 0 (zero). O máximo de acertos possíveis em cada sub-tarefa é dez e o critério de êxito individual é de, no mínimo, $50 \%$ de acertos ${ }^{(7-8)}$.

Após a coleta, os dados foram tratados estatisticamente por meio do teste não-paramétrico de Kruskal-Wallis. O nível de significância utilizado foi de $5 \%$.

\section{RESULTADOS}

No total foram avaliadas 23 crianças com DFE, porém, como se pode observar nas tabelas que seguem, à medida que aumenta o grau de dificuldade das tarefas, diminui o número de sujeitos que as realizaram. Isso se deve ao fato de que as tarefas não são aplicáveis a todas as idades da $\operatorname{amostra}^{(7-8)}$.

De acordo com o Protocolo de Tarefas de Consciência Fonológica $^{(7)}$, cada uma das tarefas é denominada com a letra T mais o número da ordem em que o teste é apresentado, por exemplo, T4, refere-se à tarefa número quatro do protocolo síntese silábica. Para identificar a sub-tarefa é acrescentado o número de sílabas, fonemas ou posição da sílaba ou fonema na palavra, no caso a sub-tarefa T4-dissílabas faz referência à sub-tarefa de síntese silábica em palavras dissílabas. Cada tarefa, e sua respectiva sigla, encontram-se dispostas no Quadro 1.

Quadro 1. Siglas do Protocolo de Tarefas em Consciência Fonológica ${ }^{(7)}$

\begin{tabular}{|c|c|c|c|}
\hline Tarefa & Sigla & Tarefa & Sigla \\
\hline Síntese silábica & $\mathrm{T} 4$ & Posição inicial & T8-inicial \\
\hline Com dissílabas & T4-dissílabas & Posição final & T8-final \\
\hline Com trissílabas & T4-trissílabas & Posição medial & T8-medial \\
\hline Com quadrissílabas & T4-quadrissílabas & Detecção fonêmica & T9 \\
\hline Segmentação silábica & T5 & Posição inicial & T9-inicial \\
\hline Com dissílabas & T5-dissílabas & Posição final & T9-final \\
\hline Com trissílabas & T5-trissílabas & Posição medial & T9-medial \\
\hline Com quadrissílabas & T5-quadrissílabas & Síntese fonêmica & $\mathrm{T} 10$ \\
\hline Detecção silábica & T6 & Com 3 fonemas & T10 - 3 fonemas \\
\hline Posição inicial & T6-inicial & Com 4 fonemas & T10 - 4 fonemas \\
\hline Posição final & T6-final & Com 5 fonemas & T10 - 5 fonemas \\
\hline Posição medial & T6-medial & Com 6 fonemas & T10 - 6 fonemas \\
\hline Reversão silábica & T7 & Segmentação fonêmica & $\mathrm{T} 11$ \\
\hline Com dissílabas & T7-dissílabas & Com 3 fonemas & T11 - 3 fonemas \\
\hline Com trissílabas & T7-trissílabas & Com 4 fonemas & T11 - 4 fonemas \\
\hline Com quadrissílabas & T7-quadrissilabas & Com 5 fonemas & T11 - 5 fonemas \\
\hline \multirow[t]{2}{*}{ Exclusão fonêmica } & T8 & Reversão fonêmica & $\mathrm{T} 12$ \\
\hline & & Com 2 e 3 fonemas & $\mathrm{T} 12-2$ e 3 fonemas \\
\hline
\end{tabular}


Na Tabela 1, é possível observar que os sujeitos tiveram facilidade na realização da tarefa de síntese silábica (T4), e que não houve diferença estatisticamente significativa entre os grupos. Tais dados se baseiam no fato de que as médias de acerto são altas e muito semelhantes. Mas, como é possível notar, as médias do GE2 em todas as tarefas são inferiores às do GE1, o que evidencia a tendência de o grupo com terapia ter melhor desempenho na tarefa T4. Outro dado interessante é que, para os sujeitos do GE1, a tarefa que pareceu ser mais fácil foi a sub-tarefa T4-trissílabas, enquanto que para o GE2 foi a sub-tarefa T4-quadrissílabas.

Os sujeitos de ambos os grupos tiveram dificuldade com a tarefa de segmentação silábica (T5), mas não houve diferenças estatisticamente significativas. Porém, o dado mais interessante desses resultados é que o GE2 teve melhor desempenho na sub-tarefa T5-dissílabas, enquanto nas demais sub-tarefas o GE1 obteve melhores resultados (Tabela 1).

Para a tarefa de detecção de sílabas, os sujeitos do GE2 tiveram pior desempenho em todas as sub-tarefas em comparação com o GE1, como se pode notar pelas médias de acertos de ambos os grupos. Para o grupo sem terapia (GE2), a sub-tarefa que mostrou ser mais difícil foi a T6-medial conforme esperado, pois, dentre as três sub-tarefas, é a que apresenta maior grau de dificuldade; porém para o grupo com terapia (GE1) a sub-tarefa mais difícil parece ser a T6-final, conforme ilustrado pelas médias de acerto.
$\mathrm{Na}$ tarefa de reversão silábica é notável o melhor desempenho do GE1 em comparação com o GE2. Na sub-tarefa T7-dissílabas houve diferença estatisticamente significativa, com desempenho melhor do GE1. Porém, as crianças que foram submetidas à terapia obtiveram médias de acerto muito baixas, ainda que essas médias sejam superiores às das crianças que não receberam intervenção fonoaudiológica. Outro dado observado é que quanto maior a quantidade de sílabas, maior a dificuldade das crianças na realização das tarefas. As mesmas não obtiveram êxito nas tarefas com trissílabas e quadrissílabas (Tabela 1).

Nenhuma das crianças do GE2 submetidas à tarefa de exclusão fonêmica obteve êxito (Tabela 2). Confirmou-se, novamente, a tendência de o GE1 ter melhor desempenho do que o GE2.

Os sujeitos do GE1 tiveram desempenhos muito baixos, principalmente na sub-tarefa T8-inicial, seguida pela T8medial e T8-final. O esperado seria que as crianças tivessem melhor desempenho em T8-inicial, seguida por T8-final e por último T8-medial. Esses resultados mostram que, apesar de receberem intervenção terapêutica, as crianças ainda têm dificuldades com as habilidades em CF, principalmente com as tarefas no nível do fonema.

$\mathrm{Na}$ tarefa de deteç̧ão fonêmica, novamente não houve diferença estatisticamente significativa. Porém, comparandose as médias, pode-se observar o melhor desempenho do

Tabela 1. Resultados das tarefas em consciência silábica para cada sub-teste

\begin{tabular}{|c|c|c|c|c|c|}
\hline Sub-tarefa & Grupos & $\mathrm{N}$ & Média & DP & Valor de $p$ \\
\hline \multirow[t]{2}{*}{ T4-dissílabas } & GE1 & 14 & 9,5714286 & 1,1578684 & 0,3820 \\
\hline & GE2 & 9 & 9,4444444 & 0,8819171 & \\
\hline \multirow[t]{2}{*}{ T4-trissílabas } & GE1 & 14 & 9,7142857 & 0,7262730 & 0,5665 \\
\hline & GE2 & 9 & 9,3333333 & 1,4142136 & \\
\hline \multirow[t]{2}{*}{ T4-quadrissílabas } & GE1 & 14 & 9,5714286 & 1,1578684 & 0,7024 \\
\hline & GE2 & 9 & 9,5555556 & 0,8819171 & \\
\hline \multirow[t]{2}{*}{ T5-dissílabas } & GE1 & 14 & 7,7142857 & 2,4314785 & 0,7633 \\
\hline & GE2 & 9 & 7,7777778 & 3,3458100 & \\
\hline \multirow[t]{2}{*}{ T5-trissílabas } & GE1 & 14 & 7,4285714 & 3,3215910 & 0,8973 \\
\hline & GE2 & 9 & 7,1111111 & 3,8550112 & \\
\hline \multirow[t]{2}{*}{ T5-quadrissílabas } & GE1 & 14 & 7,4285714 & 3,6943676 & 0,6334 \\
\hline & GE2 & 9 & 6,8888889 & 3,8550112 & \\
\hline \multirow[t]{2}{*}{ T6-inicial } & GE1 & 11 & 5,9090909 & 3,1449816 & 0,8785 \\
\hline & GE2 & 6 & 5,3333333 & 4,1793141 & \\
\hline \multirow[t]{2}{*}{ T6-final } & GE1 & 11 & 4,1818182 & 3,4005347 & 0,7613 \\
\hline & GE2 & 6 & 4,0000000 & 3,5213634 & \\
\hline \multirow[t]{2}{*}{ T6-medial } & GE1 & 11 & 5,1818182 & 2,6764970 & 0,3024 \\
\hline & GE2 & 6 & 3,5000000 & 3,2093613 & \\
\hline \multirow[t]{2}{*}{ T7-dissílabas } & GE1 & 9 & 3,8888889 & 3,7896057 & $0,0347^{*}$ \\
\hline & GE2 & 6 & 0.3333333 & 0,8164966 & \\
\hline \multirow[t]{2}{*}{ T7-trissílabas } & GE1 & 7 & 3,1428571 & 3,4364988 & 0,0635 \\
\hline & GE2 & 3 & 0 & 0 & \\
\hline \multirow[t]{2}{*}{ T7-quadrissílabas } & GE1 & 7 & 2,2857143 & 2,2146697 & 0,1220 \\
\hline & GE2 & 3 & 0 & 0 & \\
\hline
\end{tabular}

Teste Kruskal-Wallis; * Valores estatisticamente significantes $(p<0,05)$

Legenda: GE1 = grupo de estudo 1 (com terapia); GE2 = grupo de estudo 2 (sem terapia); $\mathrm{N}$ = número de sujeitos; DP = desvio-padrão 
Tabela 2. Resultados das tarefas em consciência fonêmica para cada sub-teste

\begin{tabular}{|c|c|c|c|c|c|}
\hline Sub-tarefa & Grupo & $\mathrm{N}$ & Média & $\mathrm{DP}$ & Valor de $\mathrm{p}$ \\
\hline \multirow[t]{2}{*}{ T8-inicial } & GE1 & 07 & 1,8571429 & 2,6095064 & 0,2070 \\
\hline & GE2 & 03 & 0 & 0 & \\
\hline \multirow[t]{2}{*}{ T8-final } & GE1 & 07 & 4,2857143 & 5,3452248 & 0,1985 \\
\hline & GE2 & 03 & 0 & 0 & \\
\hline \multirow[t]{2}{*}{ T8-medial } & GE1 & 07 & 3,5714286 & 4,4668088 & 0,2049 \\
\hline & GE2 & 03 & 0 & 0 & \\
\hline \multirow[t]{2}{*}{ T9-inicial } & GE1 & 11 & 4,4545455 & 3,8823610 & 0,3328 \\
\hline & GE2 & 06 & 3,0000000 & 3,5213634 & \\
\hline \multirow[t]{2}{*}{ T9-final } & GE1 & 08 & 3,0000000 & 2,7255406 & 0,5971 \\
\hline & GE2 & 05 & 2,2000000 & 3,0331502 & \\
\hline \multirow[t]{2}{*}{ T9-medial } & GE1 & 07 & 3,5714286 & 2,8784917 & 0,4043 \\
\hline & GE2 & 03 & 1,6666667 & 2,8867513 & \\
\hline \multirow[t]{2}{*}{$\mathrm{T} 10-3$ fonemas } & GE1 & 06 & 0,3333333 & 0,8164966 & 0,5930 \\
\hline & GE2 & 03 & 0,6666667 & 1,1547005 & \\
\hline \multirow[t]{2}{*}{ T10 - 4 fonemas } & GE1 & 06 & 0,3333333 & 0,8164966 & 0,4795 \\
\hline & GE2 & 03 & 0 & 0 & \\
\hline \multirow[t]{2}{*}{ T10-5 fonemas } & GE1 & 06 & 0 & 0 & 1,0000 \\
\hline & GE2 & 03 & 0 & 0 & \\
\hline \multirow[t]{2}{*}{ T10-6 fonemas } & GE1 & 05 & 0 & 0 & 1,0000 \\
\hline & GE2 & 03 & 0 & 0 & \\
\hline \multirow[t]{2}{*}{ T11 - 3 fonemas } & GE1 & 06 & 0 & 0 & 1,0000 \\
\hline & GE2 & 03 & 0 & 0 & \\
\hline \multirow[t]{2}{*}{ T11-4 fonemas } & GE1 & 06 & 0 & 0 & 1,0000 \\
\hline & GE2 & 03 & 0 & 0 & \\
\hline \multirow[t]{2}{*}{ T11 - 5 fonemas } & GE1 & 06 & 0 & 0 & 1,0000 \\
\hline & GE2 & 03 & 0 & 0 & \\
\hline \multirow[t]{2}{*}{ T12 - 2 e 3 fonemas } & GE1 & 06 & 0 & 0 & 1,0000 \\
\hline & GE2 & 03 & 0 & 0 & \\
\hline
\end{tabular}

Teste Kruskal-Wallis; * Valores estatisticamente significantes

Legenda: GE1 = grupo de estudo 1 (com terapia); GE2 = grupo de estudo 2 (sem terapia); N = número de sujeitos; DP = desvio-padrão

GE1 em relação ao GE2. No GE2, conforme aumentou a dificuldade das sub-tarefas, pior foi o desempenho. Já o GE1 obteve um desempenho pior na sub-tarefa T9-final, seguida pela T9-medial.

Para a tarefa de síntese fonêmica, observa-se que o desempenho dos sujeitos nas sub-tarefas que conseguiram realizar foi muito baixo. Na sub-tarefa T10 - 3 fonemas, o GE2 obteve média superior ao GE1. Porém, o GE2 não conseguiu realizar as demais tarefas. Tais resultados mostram a grande dificuldade que crianças com DFE têm na realização de tarefas de $\mathrm{CF}$, principalmente no nível do fonema.

Na tarefa de segmentação fonêmica, observa-se que nenhum dos sujeitos conseguiu realizar a tarefa. Tal resultado confirma a dificuldade das crianças com DFE na realização das tarefas de $\mathrm{CF}$, mesmo para aquelas que recebem terapia fonológica.

Assim como na tarefa de reversão fonêmica, os sujeitos seguem a mesma tendência anteriormente comentada. Nenhuma criança conseguiu realizar a tarefa, tanto do GE1 quanto do GE2. Esses dados confirmam a dificuldade que as crianças com fala desviante têm nas habilidades metafonológicas, principalmente no nível do fonema, independentemente da intervenção fonoaudiológica.

Resumindo os dados apresentados, foram aplicadas nove tarefas de CF em ambos os grupos, sendo divididas em vinte e seis sub-tarefas. Houve diferença estatisticamente significativa somente em uma sub-tarefa (reversão silábica,T7- dissílabas). Em outras seis sub-tarefas (síntese fonêmica T10 - 5 fonemas e T10 - 6 fonemas; segmentação fonêmica T11 - 3 fonemas, T11 - 4 fonemas e T11 - 5 fonemas; reversão fonêmica T12 - 2 e 3 fonemas) nenhum dos sujeitos pesquisados conseguiu realizá-las. Em duas sub-tarefas (segmentação silábica T5dissílabas e síntese fonêmica T10 - 3 fonemas) o GE2 obteve melhor desempenho. Nas demais sub-tarefas, houve tendência de o GE1 obter melhor desempenho, conforme a análise das médias de acerto.

\section{DISCUSSÃO}

Em relação aos resultados, pode-se observar nas tabelas que houve diferença significativa somente em uma sub-tarefa de reversão silábica com dissílabas (T7- dissílabas). Mas notase, pelas médias apresentadas, que existe uma tendência de o grupo sem terapia ter pior desempenho nas tarefas de $\mathrm{CF}$ do que 
o grupo com terapia, apesar de não haver diferença significativa entre os grupos. Os sujeitos que receberam terapia fonológica, mesmo apresentando melhor desempenho, tiveram escores baixos nas tarefas consideradas nesta pesquisa em relação aos escores esperados pela literatura ${ }^{(8)}$, para cada faixa etária.

De qualquer forma, observando-se as médias de desempenho dos sujeitos do presente estudo, pode-se inferir que a terapia fonológica traz benefícios para o DFE nas habilidades em $\mathrm{CF}$, mesmo que indiretamente. Como mostram os resultados, houve uma tendência a um melhor desempenho do GE1 em comparação ao GE2 em 15 sub-tarefas e, na sub-tarefa T7dissílabas (reversão silábica), houve diferença significativa a favor do GE1. A terapia fonológica, mesmo não abordando diretamente tarefas de $\mathrm{CF}$, proporciona à criança maior atenção para os contrastes fônicos da fala ${ }^{(5,15,20)}$, o que poderia ser considerado estímulo da habilidade metafonológica. Como já foi mencionado, o grupo que recebeu terapia também apresentou baixo desempenho nas tarefas de $\mathrm{CF}$. Esse resultado não seria esperado caso fosse assumida a hipótese deste trabalho de que os sujeitos com DFE que receberam intervenção obteriam melhora nas habilidades metafonológicas. Como os modelos terapêuticos utilizados não enfocam as habilidades metafonológicas, talvez seja necessário que tais habilidades sejam trabalhadas especificamente em terapia, para que crianças com DFE tenham um melhor desempenho em CF.

Contudo, encontram-se na literatura pesquisas que confirmam esse achado, relatando que algumas crianças com DFE somente superarão as dificuldades e desenvolverão as habilidades em CF após uma intervenção metafonológica, associada ou não à terapia fonológica ${ }^{(5,15,18,20-24)}$.

Outro estudo que corrobora os achados desta pesquisa avaliou a relação entre $\mathrm{CF}$ e habilidades em escrita em crianças com DFE, após a alta da fonoterapia. Tal estudo concluiu que essas crianças poderão apresentar dificuldades com a escrita e a $\mathrm{CF}$, mesmo submetidas à terapia ${ }^{(25)}$. As autoras ainda sugerem que as habilidades metafonológicas devem ser estimuladas nas crianças com DFE, independentemente da abordagem fonológica que se está usando.

Por outro lado, algumas pesquisas relatam que nem todas as crianças com DFE terão problemas com as habilidades necessárias para aquisição da leitura e da escrita, como as habilidades metafonológicas. Isso não foi observado neste estudo, pois a maioria dos sujeitos mostrou baixo desempenho em grande parte das tarefas de $\mathrm{CF}$ testadas ${ }^{(21-22,26)}$. As crianças com DFE têm baixo desempenho nas tarefas de CF, mas são capazes de realizar as tarefas apesar do desvio de fala.

Porém, o que parece ser consenso entre os pesquisadores é que a dificuldade em $\mathrm{CF}$ das crianças com DFE está centrada na dificuldade de acesso às representações fonológicas, seja no armazenamento ou na recuperação destas representações $^{(12,17-18,20-22,27-28)}$, o que, no entanto, se reflete em baixos escores em tarefas de CF. Nesses termos, a representação fonológica é utilizada para descrever o armazenamento da informação fonológica das palavras na memória de longo prazo e é considerada a base cognitiva para a geração das palavras faladas ${ }^{(29)}$

O DFE pode ser atribuído, em parte, à qualidade pobre das representações fonológicas, o que pode determinar a precisão das produções da criança. Além disso, faz com que a criança tenha maior dificuldade de formar novas representações fonológicas, gerando um atraso no desenvolvimento das habilidades metafonológicas. Tais representações, estando pouco ou inadequadamente especificadas, promovem dificuldades nas tarefas de compreensão e produção de fala e da $\mathrm{CF}^{(12,17,27-28)}$, como se verificou neste estudo em que os resultados mostraram uma tendência a baixo desempenho de todos os sujeitos nas tarefas de $\mathrm{CF}$, mesmo os que receberam terapia fonológica.

Entretanto, acredita-se que mais pesquisas sejam necessárias a fim de se investigar como as representações fonológicas se desenvolvem, principalmente em crianças com DFE, a fim de se compreender melhor esse tema. Podese observar que a grande maioria dos estudos investiga as consequências da terapia com base metafonológica na fala e na $\mathrm{CF}^{(14,21-22,24)}$. Alguns poucos estudos pesquisam a terapia fonológica associada à metafonológica ${ }^{(5,15,23)}$. Porém, são escassos os estudos que relatam os resultados da terapia fonológica na $\mathrm{CF}$, como é o caso da presente pesquisa. Apesar da escassez de estudos, é consenso entre alguns pesquisadores que a terapia fonoaudiológica, em casos de DFE, deve enfocar os dois aspectos, fonologia e metafonologia, para que a criança consiga um melhor desenvolvimento tanto na fala quanto nas habilidades em $\mathrm{CF}^{(5,18,20-21,23-24)}$, potencializando a relação entre elas.

A terapia metafonológica associada à terapia fonológica pode não ser suficiente em alguns casos de crianças com DFE e atraso no desenvolvimento da CF. Logo, outros aspectos que podem influenciar devem ser considerados e avaliados nessas crianças, tais como as habilidades em processamento auditivo e em memória de trabalho ${ }^{(3-4,30)}$. Influências ambientais, tais como acesso a material escrito em casa, características comportamentais e motivacionais da criança em relação à fala e aprendizagem, também devem ser consideradas ${ }^{(24)}$.

No caso do presente estudo, o tempo de terapia fonológica, em média quatro meses (com aproximadamente um atendimento semanal), parece não ter sido suficiente para melhorar o desempenho em tarefas de consciência silábica e fonêmica de crianças com DFE. Contudo, deixa-se a questão em aberto, isto é, será que um tempo maior de terapia levaria a resultados distintos desses aqui apresentados, ou seria necessária a inserção da estimulação específica das habilidades metafonológicas na terapia dos DFE?

\section{CONCLUSÕES}

Houve diferença estatisticamente significativa somente na sub-tarefa T7-dissílabas (reversão silábica), a favor das crianças com DFE que receberam intervenção fonológica.

Porém, notou-se tendência do grupo que recebeu terapia de ter melhor desempenho nas tarefas de CF; mas, mesmo assim, o grupo com terapia ainda permaneceu com desempenho abaixo do esperado nas habilidades metafonológicas.

$\mathrm{O}$ que os resultados mostram é que a criança tem um ganho ao ser submetida à terapia fonológica. Contudo, quatro meses de fonoterapia (com aproximadamente um atendimento semanal) com enfoque fonológico por si só parece não ser 
suficiente para que as crianças desenvolvam plenamente as habilidades em CF.

Como a literatura sobre o assunto pesquisado é escassa, a presente pesquisa vem a colaborar para os achados na área, no sentido de propor e embasar novas pesquisas e abordagens com crianças que tenham DFE. Acredita-se que uma investigação sobre a fala de crianças com DFE, que receberam alta da terapia fonológica, seja interessante para verificar qual é o papel e o tempo da abordagem fonológica para a criança desenvolver as habilidades metafonológicas, atingindo níveis esperados de desempenho.

Além disso, sugere-se a realização de estudos sobre como as representações fonológicas se desenvolvem em crianças com fala desviante, a fim de possibilitar uma intervenção mais precisa e auxiliar o processo terapêutico.

\begin{abstract}
Purpose: To compare the performances of children with evolutive phonological disorder (EPD) that had phonologically-focused speech therapy and children with EPD who did not undergo therapy in phonological awareness (PA) abilities. Methods: Twenty three subjects with EPD diagnosis were evaluated. The 14 children that composed Study Group 1 (SG1) received speech therapy, while the other nine who were part of Study Group 2 (SG2) had not yet received speech therapy. All subjects underwent speech and language pathology evaluation and PA assessment. Results: From the 26 sub-tasks of PA applied, only one showed statistically significant difference, confirming worse performance of the SG2 ( $\mathrm{p} \leq 0.05)$. However, analysis of the means showed that SG1 tended to present a better performance overall than SG2. Despite these results, even subjects who received speech therapy intervention presented low performances on the tasks. Conclusion: Statistically significant difference was observed only in the sub-test T7-two syllables (syllabic reversion) among children with EPD who received speech intervention and children with EPD without speech therapy. However, the group who did attend speech therapy showed a tendency to achieve better performance in the PA tasks.
\end{abstract}

Keywords: Speech disorders/therapy; Child language; Language development disorders/therapy; Child language; Speech therapy

\section{REFERÊNCIAS}

1. Mota HB. Terapia fonoaudiológica para os desvios fonológicos. Rio de Janeiro: Revinter; 2001.

2. Lamprecht RR, Bonilha G, Freitas GC, Matzenauer C, Mezzomo C, Oliveira $\mathrm{C}$, et al. Aquisição fonológica do português: perfil de desenvolvimento e subsídios para terapia. Porto Alegre, ARTMED; 2004. Sobre os desvios fonológicos; p.193-212.

3. Vieira MG, Mota HB, Keske-Soares M. Relação entre idade, grau de severidade do desvio fonológico e consciência fonológica. Rev Soc Bras Fonoaudiol. 2004;9(3):144-50.

4. Linassi LZ, Keske-Soares M, Mota HB. Habilidades de memória de trabalho e grau de severidade do desvio fonológico. Pró-Fono. 2005; 17(3):383-92.

5. Spíndola RA, Payão LM, Bandini HH. Abordagem fonoaudiológica em desvios fonológicos fundamentada na hierarquia dos traços distintivos e na consciência fonológica. Rev CEFAC. 2007;9(2):180-189.

6. Menezes G, Lamprecht RR. A consciência fonológica na relação falaescrita em crianças com desvios fonológicos evolutivos (DFE). Letras de Hoje. 2001;36(3):743-9.

7. Cielo CA. Habilidades em consciência fonológica em crianças de 4 a 8 anos de idade. Pró-Fono. 2002;14(3):301-12.

8. Cielo CA. Habilidades em consciência fonológica em crianças de 4 a 8 anos de idade [tese]. Porto Alegre: Pontifica Universidade Católica do Rio Grande do Sul; 2001.

9. Salgado C, Capellini SA. Desempenho em leitura e escrita de escolares com transtornos fonológicos. Psicol Esc Educ. 2004;8(2):179-88.

10. Pestun MS. Consciência fonológica no início da escolarização e o desempenho ulterior em leitura e escrita: estudo correlacional. Estud Psicol (Natal). 2005;10(3):407-12.

11. Rvachew S, Grawburg M. Correlates of phonological awareness in preschoolers with speech sound disorders. J Speech Lang Hear Res. 2006;49(1):74-87.

12. Nathan L, Stackhouse J, Goulandris N, Snowling MJ. The development of early literacy skills among children with speech difficulties: a test of the «critical age hypothesis». J Speech Lang Hear Res. 2004;47(2):377-91.
13. Carroll JM, Snowling MJ. Language and phonological skills in children at high risk of reading difficulties. J Child Psychol Psychiatry. 2004;45(3):631-40.

14. Laing SP, Espeland W. Low intensity phonological awareness training in a preschool classroom for children with communication impairments. J Commun Disord. 2005;38(1):65-82.

15. Ardenghi LG, Mota HB, Keske-Soares M. A terapia metaphon em casos de desvios fonológicos. Rev Soc Bras Fonoaudiol. 2006;11(2):106-15.

16. Freitas GC. Sobre a consciência fonológica In: Lamprecht RR, organizador. Aquisição fonológica do português: perfil de desenvolvimento e subsídios para terapia. Porto Alegre: Artmed; 2004. p. 177-92.

17. Sutherland D, Gillon GT. Assessment of phonological representations in children with speech impairment. Lang Speech Hear Serv Sch. 2005;36(4):294-307.

18. Rvachew S. Phonological processing and reading in children with speech sound disorders. Am J Speech Lang Pathol. 2007;16(3):260-70.

19. Yavas M, Hernandorena CL, Lamprecht RR. Avaliação fonológica da criança: reeducação e terapia. Porto Alegre: Artes Médicas; 1991.

20. Major EM, Bernhardt BH. Metaphonological skills of children with phonological disorders before and after phonological and metaphonological intervention. Int J Lang Commun Disord. 1998;33(4):413-44.

21. Gillon GT. The efficacy of phonological awareness intervention for children with spoken language impairment. Lang Speech Hear Serv Sch. 2000;31:126-41.

22. Gillon GT. Facilitating phoneme awareness development in 3- and 4-year-old children with speech impairment. Lang Speech Hear Serv Sch. 2005;36:308-24.

23. Bernhardt B, Major E. Speech, language and literacy skills 3 years later: a follow-up study of early phonological and metaphonological intervention. Int J Lang Commun Disord. 2005;40(1):1-27.

24. Kirk C, Gillon GT. Longitudinal effects of phonological awareness intervention on morphological awareness in children with speech impairment. Lang Speech Hear Serv Sch. 2007;38(4):342-52. 
25. Mota HB, Melo Filha MG, Lasch SS. A consciência fonológica e o desempenho na escrita sob ditado de crianças com desvio fonológico após realização de terapia fonoaudiológica. Rev CEFAC. 2007;9(4):47782.

26. Holm A, Farrier F, Dodd B. Phonological awareness, reading accuracy and spelling ability of children with inconsistent phonological disorder. Int J Lang Commun Disord. 2008;43(3):300-22.

27. Rvachew S, Chiang PY, Evans N. Characteristics of speech errors produced by children with and without delayed phonological awareness skills. Lang Speech Hear Serv Sch. 2007;38(1):60-71.
28. Sutherland D, Gillon GT. Development of phonological representations and phonological awareness in children with speech impairment. Int J Lang Commun Disord. 2007;42(2):229-50.

29. Stackhouse J, Wells B. Children's speech and literacy difficulties: a psycholinguistic framework. London: Whurr; 1997.

30. Neves IF, Shochat E. Maturação do processamento auditivo em crianças com e sem dificuldades escolares. Pró-Fono. 2005;17(3):311-20. 